\title{
Decompressive Craniectomy for Posterior Reversible Encephalopathy Syndrome (PRES): Case Report
}

\author{
Amit Azriel ${ }^{1 *}$, Israel Melamed1\#, Ilan Shelef', Yuval Sufaro3 ${ }^{3}$, Micky Gidon', \\ Vladimir Merkin1, Alexandr Shtreizent1, Avi Cohen'1 \\ ${ }^{1}$ Department of Neurosurgery, Soroka Medical Center, Be'er-Sheva, Israel \\ ${ }^{2}$ Diagnostic Imaging Institute, Soroka Medical Center, Be'er-Sheva, Israel \\ ${ }^{3}$ Department of Neurosurgery, The Royal Melbourne Hospital, Melbourne, Australia \\ Email: ${ }^{*}$ amitazriel@post.bgu.ac.il
}

Received 19 April 2015; accepted 18 May 2015; published 20 May 2015

Copyright (C) 2015 by authors and Scientific Research Publishing Inc.

This work is licensed under the Creative Commons Attribution International License (CC BY). http://creativecommons.org/licenses/by/4.0/

(c) (i) Open Access

\begin{abstract}
Background: Posterior reversible encephalopathy syndrome (PRES) is an uncommon clinicalneuroradiological syndrome with an unclear pathophysiology. Correlation between PRES and the use of immunosuppressant drugs have previously been described, as well as correlation between elevated blood pressure and PRES. Characteristic brain MRI manifestations include hyperintense lesions on T2 and FLAIR (fluid-attenuated inversion recovery) images. PRES is usually reversible within a short period of time after discontinuation of the presumably offending drug. Some cases of PRES might complicate with intracranial hemorrhage, refractory status epilepticus or expansive vasogenic brain edema (also regarded as "tumefactive" PRES). Methods: We present a case of a young man diagnosed with Hodgkin's lymphoma, following a laparotomy due to cecal volvulus. The patient received glucocorticoids and elevated blood pressure values were recorded. Brain imaging studies were performed due to generalized epileptic seizures, demonstrating neuroradiological findings consistent with PRES. Neurological and neuroradiological deterioration was noted, necessitating urgent neurosurgical intervention. A complete neurological and functional rehabilitation was achieved. Conclusion: The uncommon cases of complicated PRES should be taken under consideration whenever clinical deterioration is noted following the diagnosis of PRES. Early neuroradiological evaluation should be sought, together with aggressive medical and surgical treatment in cases of life threatening mass effect.
\end{abstract}

\footnotetext{
"Corresponding author.

\#Amit Azriel and Dr. Israel Melamed have contributed equally to this paper.
} 


\section{Keywords}

\section{PRES, Posterior Reversible Encephalopathy Syndrome, Brain Edema, Craniectomy}

\section{Background}

Reversible posterior leukoencephalopathy syndrome was first described in 1996 by Hinchey et al. as a reversible clinical-neuroradilogical syndrome [1]. Casey et al. proposed the name Posterior Reversible Encephalopathy Syndrome (PRES) in 2000 after demonstrating substantial cortical involvement on MRI FLAIR images, mainly in milder cases [2].

The pathophysiology of PRES remains unclear. Few theories were proposed, describing this syndrome as the result of disordered cerebral autoregulation and endothelial damage. Autoregulation ensures constant blood flow to the brain despite systemic blood pressure alterations by means of arteriolar constriction and dilatation. Once autoregulation breaks, regions of both vasoconstriction and vasodilatation (the sausage-string pattern) develop, resulting in brain hyperperfusion and endothelial damage [3]. The endothelial damage alters the normal blood-brain barrier function, resulting in fluid leakage and vasogenic edema [4]. Cytotoxic edema may also occur, following ischemic damage due to the vasoconstriction or as a result of the vasogenic edema itself [5].

Strong correlation was suggested between PRES and the use of many immunosuppressant drugs, such as cyclosporine that has been related to this entity since it was first described in 1996 [6], presumably due to the endothelial damage caused by these drugs. However, PRES following glucocorticoid treatment is believed to evolve indirectly mainly due to hypertension as an adverse effect [7] [8].

Acute volume overload by blood transfusion may exceed the capacity of autoregulation resulting in vasogenic edema and development of PRES [9].

The most common clinical presentation of PRES is seizures (70\% - 80\%), followed by encephalopathy, headache and visual disturbances [10]. Comorbid conditions include hypertension, renal disease, dialysis dependency, malignancy and transplantation [11] [12].

The typical neuroraiological MRI manifestations of PRES include T2 and fluid-attenuated inversion recovery (FLAIR) hyperintense vasogenic edema involving cortical and/or subcortical white matter of the affected regions, almost always involving the parieto-occipital lobes [13]. However, frontal and temporal lobes involvement is also common. Cerebellar involvement has also been described [14]. Diffusion-weighted magnetic resonance imaging (DWI) may help to discriminate between vasogenic and cytotoxic edema, and apparent diffusion coefficient (ADC) mapping can be useful to rule out other conditions that might mimic PRES, such as central pontine myelinolysis [6].

PRES is usually fully reversible within a period of days to weeks, after removal of the offending drug and control of the elevated blood pressure. In the majority of PRES cases complete or near complete resolution of brain edema was noted during neuroimaging follow-up [15].

Intracranial hemorrhage might occur in PRES cases, mostly among patients receiving anticoagulation therapy [16]. Expansive vasogenic edema with intracranial mass effect might occur in less than $5 \%$ of cases, regarded as "tumefactive” PRES [12]. Severe and hemorrhagic PRES variants may obligate urgent life-saving neurosurgical intervention designed to address intracranial hypertension, such as invasive ICP monitoring, CSF diversion procedures or decompressive craniectomy [17].

Here we report a case of a young man who diagnosed with Hodgkin's lymphoma that developed tumefactive PRES and required lifesaving decompressive craniectomy.

\section{Case Report}

A 25-year-old man with no known chronic diseases was admitted to the emergency room at our medical institute after a day of severe abdominal pain. On admission the patient looked cachectic and reported few months of decreased appetite and significant weight loss. Clinical and radiological assessment raised suspicion for cecal volvulus and urgent laparotomy was performed, confirming the diagnosis. A cervical lymph node biopsy was also performed due to significant lymphadenopathy. The diagnosis of Hodgkin's lymphoma was established and medical treatment with glucocorticoids was initiated. Second laparotomy was performed following general and 
hemodynamic deterioration, requiring vasopressors support for a short period of time. Fecal peritonitis was diagnosed, necessitating four additional laparotomies. Elevated blood pressure values were recorded. Following a generalized epileptic seizure treatment with phenytoin was initiated. Brain CT scan demonstrated bilateral cerebellar hypodense lesions without significant mass effect (Figure 1). Brain MRI demonstrated hyperintese cortical and subcortical lesions in the temporal and occipital lobes of both cerebral hemispheres, as well as in the right frontal lobes and the cerebellum, consistent with the diagnosis of PRES (Figure 2). Epileptic seizures were controlled, and the patient regained full consciousness. ABVD chemotherapy protocol (Adriamycin, Bleomycin, Vinblastine, Dacarbazine) was initiated. Rapid neurological deterioration was noted few days later, with recurrent epileptic seizures and decline in the patient's level of consciousness. Second brain MRI (5 days following the previous one) demonstrated significant intracranial mass effect in the right cerebral hemisphere which was consistent with tumefactive PRES (Figure 3). An urgent right decompressive craniectomy was performed. Following the operation the patient recovered well with improvement of consciousness and complete seizures control. He was discharged after a short period of time with left hemiparesis to continue chemotherapy. Three months late, on outpatient clinic follow-up examination, the patient had no motor impairment and was scheduled for a skull reconstruction operation.

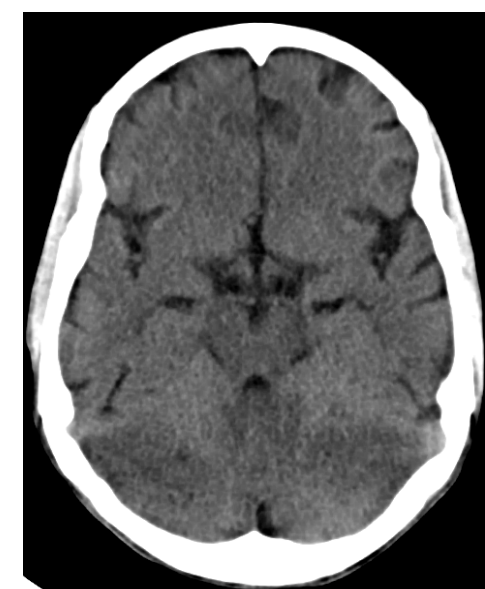

(a)

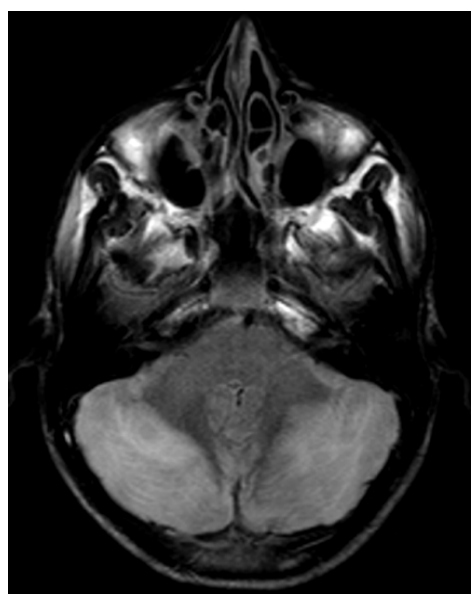

(b)

Figure 1. Bilateral cerebellar hypodense lesions on CT scan (a) and hyperintense on MRI-FLAIR (b).

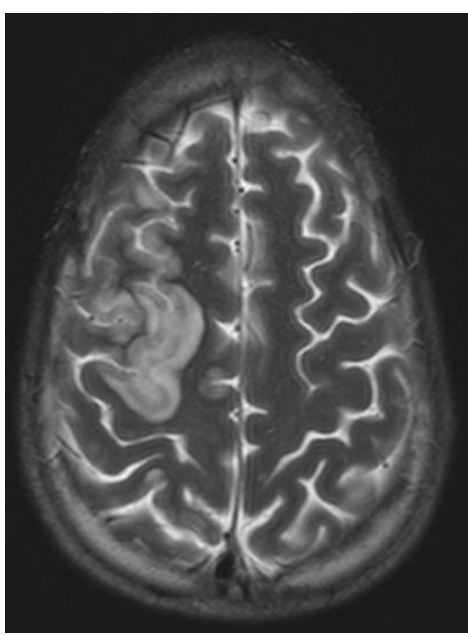

(a)

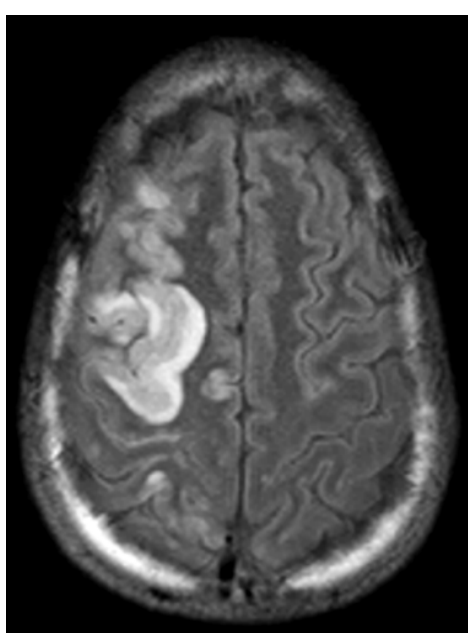

(b)

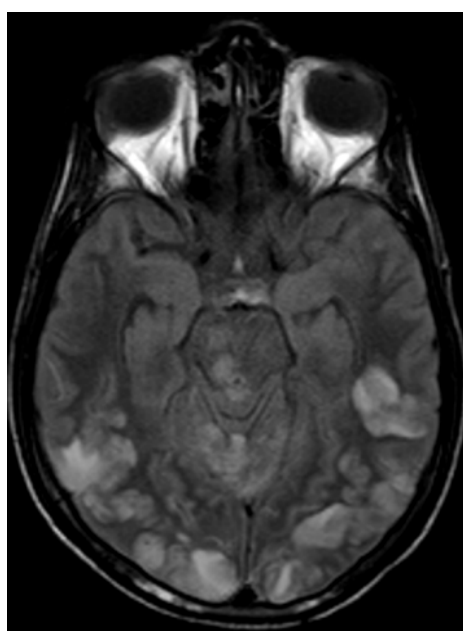

(c)

Figure 2. Brain MRI demonstrating areas of high signal involving the right frontal lobe ((a): T2 sequence), bilateral temporal and occipital lobes ((b) and (c): MRI-FLAIR). 


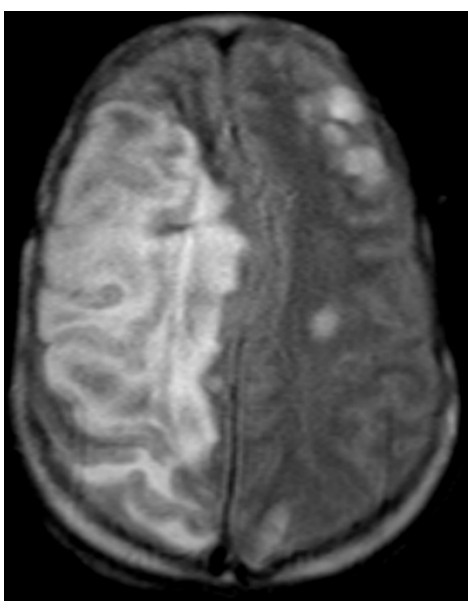

(a)

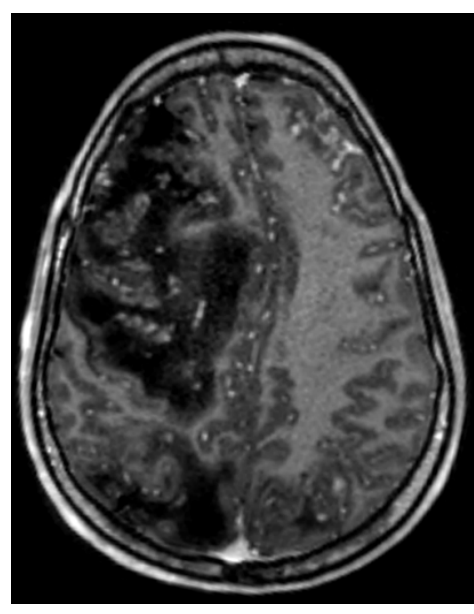

(b)

Figure 3. Second MRI examination demonstrating diffuse pathological signal with severe mass effect in the right cerebral hemisphere ((a) FLAIR; (b) T1 with gadolinium).

\section{Discussion}

PRES is considered as a benign, self-limiting disease. Complications of PRES include intracranial hemorrhage, brain edema and refractory status epilepticus. Hefzy et al. retrospectively assessed 151 patients diagnosed with PRES and identified an overall risk of 15.2\% for intracranial hemorrhage [16]. Of the 23 patients diagnosed with intracranial hemorrhage, seven (30\%) had poor clinical outcome with overall mortality rate of $26 \%$. Hemorrhage was significantly more common after allogeneic bone marrow transplantation.

Akins et al. defined PRES as "malignant" [17] based on clinical assessment (GCS of less than 8 and clinical decline despite standard medical management for elevated intracranial pressure) and radiographic criteria (edema with associated mass effect; brain hemorrhage exerting mass effect; effacement of basal cisterns, transtentorial, tonsillar, or uncal herniation). Five cases of malignant PRES were identified during a four-year period, out of 4070 admissions to the neurointensive care unit. Four of them suffered from intra-parenchymal brain hemorrhage and one from hemispheral and brainstem edema. After initial trial of non-surgical management, eventually all five patients underwent craniectomy with no cases of short-term mortality.

Life threatening PRES complications have also been described among the pediatric population. Cordelli et al. stratified 27 children with PRES diagnosis during a limited period of time at their medical institution [18]. Five cases (18\%) of life-threatening complication were identified (2 cases of cerebral hemorrhage, 2 cases of diffuse brain edema and 1 case of refractory status epilepticus) with mortality rate of $40 \%$.

Nagaoka et al. described a case of PRES in a 3-year-old girl with congenital nephrotic syndrome. The PRES was accompanied by striking swelling of brain stem and tentorial herniation and was needed for performing emergency decompressive craniectomy. The patient was recovered to the previous state before onset of PRES. The authors concluded that in severe PRES with unstable vital signs, surgical intervention should be considered, as well as appropriate blood pressure management [12].

Other medical conditions may mimic PRES and should be therefore taken under consideration when establishing the diagnosis. Subacute bilateral infarcts within the posterior distribution could be misinterpreted as PRES, as well as cortical edema of acute hypoxic-ischemic encephalopathy (HIE), central or extrapontine myelinolysis, infectious conditions, chronic white matter changes, brain neoplasms and acute hepatic encephalopathy [11] [12].

Our patient presented with a characteristic clinical background of corticosteroid therapy and documented elevated blood-pressure, together with generalized epileptic seizures. MRI studies demonstrated typical occipital, parietal, temporal and cerebellar T2 and FLAIR hyperintense lesions, as well as high signal on DWI, all consistent with the diagnosis of PRES. Rapid neurological deterioration with recurrent epileptic seizures and decline in the patient's level of consciousness, accompanied by tumefactive PRES on second brain MRI, required an urgent right decompressive craniectomy. Short time after operation the patient recovered with improvement of 
consciousness and complete seizures control. He completed chemotherapy and scheduled for a skull reconstruction operation without any neurological deficits.

\section{Conclusion}

PRES is not a common clinical syndrome; it should be taken under consideration with the proper anamnestic, clinical and radiological findings. Tumefactive PRES is even more uncommon, necessitating aggressive medical and neurosurgical treatment when suspected, in order to reduce mortality and improve the neurological and functional outcome.

\section{References}

[1] Hinchey, J., Chaves, C., Appignani, B., Breen, J., Pao, L., Wang, A., Pessin, M.S., Lamy, C., Mas, J.L. and Caplan, L.R. (1996) A Reversible Posterior Leucoencephalopathy Syndrome. The New England Journal of Medicine, 334, 494-500. http://dx.doi.org/10.1056/NEJM199602223340803

[2] Casey, S.O., Sampaio, R.C., Michel, E. and Truwit, C.L. (2000) Posterior Reversible Encephalopathy Syndrome: Utility of Fluid-attenuated Inversion Recovery MR Imaging in the Detection of Cortical and Subcortical Lesions. American Journal of Neuroradiology, 21, 1199-1206.

[3] Lamy, C., Oppenheim, C., Meder, J.F. and Mas, J.L. (2004) Neuroimaging in Posterior Reversible Encephalopathy Syndrome. Journal of Neuroimaging , 14, 89-96.

[4] Marra, A., Vargas, M., Striano, P., Del Guercio, L., Buonanno, P. and Servillo, G. (2014) Posterior Reversible Encephalopathy Syndrome: The Endothelial Hypotheses. Medical Hypotheses, 82, 619-622. http://dx.doi.org/10.1016/j.mehy.2014.02.022

[5] Heo, K., Park, S.A., Lee, J.Y., Lee, B.I. and Lee, S.K. (2003) Post-Transfusion Posterior Leukoencephalopathy with Cytotoxic and Vasogenic Edema Precipitated by Vasospasm. Cerebrovascular Diseases, 15, 230-233. http://dx.doi.org/10.1159/000068825

[6] Pedraza, R., Marik, P.E. and Varon, J. (2009) Posterior Reversible Encephalopathy Syndrome: A Review. Critical Care and Shock, 12, 135-143.

[7] Irvin, W., MacDonald, G., Smith, J.K. and Kim, W.Y. (2007) Dexamethasone-Induced Posterior Reversible Encephalopathy Syndrome. Journal of Clinical Oncology, 25, 2484-2486. http://dx.doi.org/10.1200/JCO.2007.10.9991

[8] Hansberry, D., Agarwal, N., Tomei, K. and Goldstein, I. (2013) Posterior Reversible Encephalopathy Syndrome in a Patient with a Chiari I Malformation. Surgical Neurology International, 4, 130.

[9] Wada, K., Kano, M., Machida, Y., Hattori, N. and Miwa, H. (2013) Posterior Reversible Encephalopathy Syndrome Induced after Blood Transfusion for Severe Anemia. Case Reports in Clinical Medicine, 5, 332-334.

[10] Fugate, J.E., Claassen, D.O., Cloft, H.J., Kallmes, D.F., Kozak, O.S. and Rabinstein, A.A. (2010) Posterior Reversible Encephalopathy Syndrome: Associated Clinical and Radiologic Findings. Mayo Clinic Proceedings, 85, 427-432. http://dx.doi.org/10.4065/mcp.2009.0590

[11] Lee, V.H., Wijdicks, E.F.M., Manno, E.M. and Rabinstein, A.A. (2008) Clinical Spectrum of Reversible Posterior Leukoencephalopathy Syndrome. Archives of Neurology, 65, 205-210.

[12] Nagaoka, Y., Ishikura, K., Hamada, R., Miyagawa, T., Kono, T., Sakai, T., Hamasaki, Y., Hataya, H. and Honda, M. (2013) Severe Posterior Reversible Encephalopathy Syndrome Resolved with Craniectomy. Pediatrics International, 55, 644-646. http://dx.doi.org/10.1111/ped.12084

[13] Rykken, J.B. and McKinney, A.M. (2014) Posterior Reversible Encephalopathy Syndrome. Seminars in Ultrasound, CT, and MRI, 35, 118-135.

[14] Bartynski, W.S. (2008) Posterior Reversible Encephalopathy Syndrome, Part 1: Fundamental Imaging and Clinical Features. American Journal of Neuroradiology, 29, 1036-1042. http://dx.doi.org/10.3174/ajnr.A0928

[15] Liman, T.G., Bohner, G., Heuschmann, P.U., Endres, M. and Siebert, E. (2012) The Clinical and Radiological Spectrum of Posterior Reversible Encephalopathy Syndrome: The Retrospective Berlin PRES Study. Journal of Neurology, 259, 155-164. http://dx.doi.org/10.1007/s00415-011-6152-4

[16] Hefzy, H.M., Bartynski, W.S., Boardman, J.F. and Lacomis, D. (2009) Hemorrhage in Posterior Reversible Encephalopathy Syndrome: Imaging and Clinical Features. American Journal of Neuroradiology, 30, 1371-1379.

[17] Akins, P.T., Axelrod, Y., Silverthorn, J.W., Guppy, K., Banerjee, A. and Hawk, M.W. (2014) Management and Outcomes of Malignant Posterior Reversible Encephalopathy Syndrome. Clinical Neurology and Neurosurgery, 125, 5257. http://dx.doi.org/10.1016/j.clineuro.2014.06.034 
[18] Cordelli, D.M., Masetti, R., Ricci, E., Toni, F., Zama, D., Maffei, M., Gentili, A., Parmeggiani, A., Pession, A. and Franzoni, E. (2014) Life-Threatening Complications of Posterior Reversible Encephalopathy Syndrome in Children. European Journal of Pediatric Neurology, 18, 632-640. http://dx.doi.org/10.1016/j.ejpn.2014.04.014 